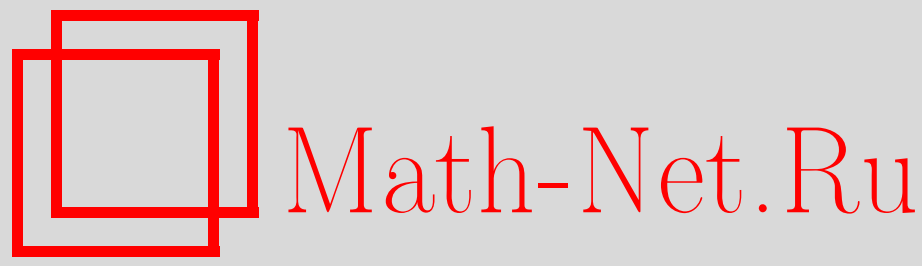

В. В. Жаринов, Эволюционные системы со связями в виде условий нулевой дивергенции, ТМФ, 2010, том 163, номер 1, 3-16

DOI: https://doi.org/10.4213/tmf6483

Использование Общероссийского математического портала Math-Net.Ru подразумевает, что вы прочитали и согласны с пользовательским соглашением http://www.mathnet.ru/rus/agreement

Параметры загрузки:

IP : 54.196 .121 .252

26 апреля 2023 г., 14:46:54

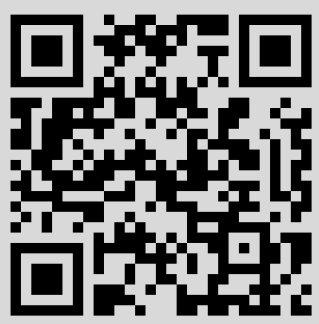




\section{ЭВОЛЮЦИОННЫЕ СИСТЕМЫ СО СВЯЗЯМИ В ВИДЕ УСЛОВИЙ НУЛЕВОЙ ДИВЕРГЕНЦИИ}

Изучаются эволюционные системы уравнений в частных производных при наличии согласованных связей в виде системы уравнений неразрывности. Показано, что всякая такая система наряду с возможными законами сохранения стандартной степени, равной числу пространственных переменных, обладает и законами сохранения низшей степени, на единицу меньшей числа пространственных переменных. Предварительно дано полное описание законов сохранения и симметрий системы уравнений неразрывности. В качестве примера вычислены законы сохранения второй степени классической системы уравнений Максвелла (здесь число пространственных переменных равно трем).

Ключевые слова: эволюционные системы, связи, уравнения неразрывности, законы сохранения, законы сохранения низшей степени.

\section{1. ВВЕДЕНИЕ}

Известно, что эволюционные системы вида

$$
\partial_{t} u=F\left(t, x, u, u_{x}, u_{x x}, \ldots\right),
$$

где $x \in \mathbb{R}^{m}$ - пространственные переменные, $u \in \mathbb{R}^{N}$ - зависимые переменные, $u_{x}-$ все частные производные первого порядка по $x, u_{x x}$ - все частные производные второго порядка по $x$ и т. д., могут обладать законами сохранения лишь степени $m$ (при их записи в виде дифференциальных форм, замкнутых на решениях). В предлагаемой работе изучаются эволюционные системы вида (1.1) при наличии согласованных связей в виде системы уравнений неразрывности

$$
\sum_{1 \leqslant \mu \leqslant m} \partial_{x^{\mu}} u^{\sigma \mu}=0, \quad 1 \leqslant \sigma \leqslant s .
$$

Здесь $N=s m$; зависимые переменные $u \in \mathbb{R}^{s m}$ маркируются двойным индексом $\sigma \mu$. Показано, что в этом случае объединенная система (1.1), (1.2) всегда обладает законами сохранения низшей степени $m-1$, причем базисные законы сохранения

* Математический институт им. В. А. Стеклова РАН, Москва, Россия. E-mail: zharinov@mi.ras.ru 
выписаны в явном виде. Предварительно проведен алгебро-геометрический анализ системы (1.2) и получено полное описание ее законов сохранения и симметрий. В качестве примера в явном виде вычислены законы сохранения низшей степени классической системы уравнений Максвелла.

\section{2. АЛГЕБРО-ГЕОМЕТРИЧЕСКИЙ АНАЛИЗ УРАВНЕНИЙ НЕРАЗРЫВНОСТИ}

2.1. Обозначения. Будем использовать следующие обозначения:

- $\mathbb{Z}_{+}=\{0,1,2, \ldots\}$ - множество всех неотрицательных целых чисел;

- $\mathbb{N}=\{1,2,3, \ldots\}$ - множество всех натуральных чисел;

- $x=\left(x^{\mu} \mid 1 \leqslant \mu \leqslant m\right) \in \mathbb{R}^{m}$ - независимые переменные;

- $u=\left(u^{\sigma \mu} \mid 1 \leqslant \sigma \leqslant s, 1 \leqslant \mu \leqslant m\right) \in \mathbb{R}^{s m}$ - зависимые переменные;

- $i=\left(i^{\mu} \mid 1 \leqslant \mu \leqslant m\right) \in \mathbb{Z}_{+}^{m}-$ мультииндексы;

- $|i|=i^{1}+\cdots+i^{m}, i+j=\left(i^{\mu}+j^{\mu}\right),(\nu)=(0, \ldots, 1, \ldots, 0)$ - единица стоит на $\nu$-м месте, в частности, $i+(\nu)=\left(i^{1}, \ldots, i^{\nu}+1, \ldots, i^{m}\right)$;

- $\mathbf{u}=\left(u_{i}^{\sigma \mu} \mid 1 \leqslant \sigma \leqslant s, 1 \leqslant \mu \leqslant m, i \in \mathbb{Z}_{+}^{m}\right) \in \mathbb{R}_{\mathbb{Z}_{+}^{m}}^{s m}$ - дифференциальные переменные, $u^{\sigma \mu}=u_{0}^{\sigma \mu}$;

- $\mathfrak{A}=\mathcal{C}_{\mathrm{fin}}^{\infty}\left(\mathbb{R}^{m} \times \mathbb{R}_{\mathbb{Z}_{+}^{m}}^{s m}\right)$ - алгебра дифференииалъных функиий, т. е. всех вещественных гладких (класса $\mathcal{C}^{\infty}$ ) функций $f=f(x, \mathbf{u})$ конечного порядка, т. е. зависящих лишь от конечного числа аргументов;

- $\partial_{x^{\mu}}, \partial_{u^{\sigma \mu}}, \partial_{u_{i}^{\sigma \mu}}, \ldots$ - частные производные по $x^{\mu}, u^{\sigma \mu}, u_{i}^{\sigma \mu}, \ldots$;

- $D_{\mu}=\partial_{x^{\mu}}+\sum_{\sigma, \nu, i} u_{i+(\mu)}^{\sigma \nu} \partial_{u_{i}^{\sigma \nu}}, 1 \leqslant \mu \leqslant m,-$ полные частные производные.

2.2. Уравнения неразрывности. Первый этап изучения эволюции с нулевой дивергенцией есть алгебро-геометрический анализ уравнений связи, в нашем случае - уравнений неразрывности

$$
\sum_{1 \leqslant \mu \leqslant m} \partial_{x^{\mu}} u^{\sigma \mu}=0, \quad 1 \leqslant \sigma \leqslant s .
$$

2.3. Уравнения неразрывности как эволюционная система. Положим $u_{i}^{\sigma \mu}=\partial_{x^{i}} u^{\sigma \mu}$, где $\partial_{x^{i}}=\partial_{x^{1}}^{i^{1}} \ldots \partial_{x^{m}}^{i^{m}}$. Тогда система (2.1) плюс все ее дифференциальные продолжения запишутся в виде

$$
\sum_{1 \leqslant \mu \leqslant m} u_{i+(\mu)}^{\sigma \mu}=0, \quad 1 \leqslant \sigma \leqslant s, \quad i \in \mathbb{Z}_{+}^{m} .
$$

Согласно стандартным правилам алгебро-геометрического анализа дифференциальных уравнений (см., например, работы [1]-[6]) бесконечная система уравнений (2.2) определяет гладкую поверхность

$$
\mathcal{X}=\left\{(x, \mathbf{u}) \mid \sum_{1 \leqslant \mu \leqslant m} u_{i+(\mu)}^{\sigma \mu}=0,1 \leqslant \sigma \leqslant s, i \in \mathbb{Z}_{+}^{m}\right\}
$$

в пространстве $\mathbb{R}^{m} \times \mathbb{R}_{\mathbb{Z}_{+}^{m}}^{s m}$ с базисными картановыми дифференцированиями $\partial_{\mu}=$ $\left.D_{\mu}\right|_{\mathcal{X}}, 1 \leqslant \mu \leqslant m$. Глобальные координаты на поверхности $\mathcal{X}$ суть независимые 
переменные $x=\left(x^{\mu} \mid 1 \leqslant \mu \leqslant m\right) \in \mathbb{R}^{m}$ и свободные дифференциальные переменные $\left(u_{l}^{\sigma}, u_{\kappa l}^{\sigma \alpha}\right) \in \mathbb{R}_{\mathbb{Z}_{+}^{m-1}}^{s} \times \mathbb{R}_{\mathbb{Z}_{+}^{m}}^{s(m-1)}$, где $1 \leqslant \sigma \leqslant s, 2 \leqslant \alpha \leqslant m, \kappa \in \mathbb{Z}_{+}$, индексы $l=\left(l^{\alpha} \mid 2 \leqslant\right.$ $\alpha \leqslant m) \in \mathbb{Z}_{+}^{m-1}, i=\kappa l \in \mathbb{Z}_{+}^{m}, u_{l}^{\sigma}=u_{0 l}^{\sigma 1}$. Остальные дифференциальные переменные находятся из системы уравнений $(2.2)$ :

$$
u_{\kappa l}^{\sigma 1}=-\sum_{2 \leqslant \alpha \leqslant m} u_{\kappa-1, l+(\alpha)}^{\sigma \alpha}, \quad 1 \leqslant \sigma \leqslant s, \quad \kappa \in \mathbb{N}, \quad l \in \mathbb{Z}_{+}^{m-1} .
$$

Алгебра дифференциальных функций на поверхности $\mathcal{X}$ есть алгебра $\mathcal{A}=$ $\mathcal{C}_{\text {fin }}^{\infty}\left(\mathbb{R}^{m} \times \mathbb{R}_{\mathbb{Z}_{+}^{s-1}}^{s} \times \mathbb{R}_{\mathbb{Z}_{+}^{m}}^{s(m-1)}\right)$ всех вещественных гладких функций конечного порядка на пространстве $\mathbb{R}^{m} \times \mathbb{R}_{\mathbb{Z}_{+}^{s-1}}^{s} \times \mathbb{R}_{\mathbb{Z}_{+}^{m}}^{s(m-1)}$, базисные картановы дифференцирования записываются в виде

$$
\begin{aligned}
& \partial_{1}=\partial_{x^{1}}+\sum_{\sigma, l} U_{1 l}^{\sigma 1} \partial_{u_{l}^{\sigma}}+\sum_{\sigma, \beta, \kappa l} u_{\kappa+1, l}^{\sigma \beta} \partial_{u_{\kappa l}^{\sigma \beta}}, \quad U_{1 l}^{\sigma 1}=-\sum_{2 \leqslant \beta \leqslant m} u_{0, l+(\beta)}^{\sigma \beta}, \\
& \partial_{\alpha}=\partial_{x^{\alpha}}+\sum_{\sigma, l} u_{l+(\alpha)}^{\sigma} \partial_{u_{l}^{\sigma}}+\sum_{\sigma, \beta, \kappa l} u_{\kappa, l+(\alpha)}^{\sigma \beta} \partial_{u_{\kappa l}^{\sigma \beta}}, \quad 2 \leqslant \alpha \leqslant m .
\end{aligned}
$$

Заметим, что $u_{l}^{\sigma}=\partial_{l} u^{\sigma}, u_{\kappa l}^{\sigma \alpha}=\partial_{l} u_{\kappa}^{\sigma \alpha}, 1 \leqslant \sigma \leqslant s, 2 \leqslant \alpha \leqslant m, \kappa \in \mathbb{Z}_{+}$, где $l=$ $\left(l^{2}, \ldots, l^{m}\right) \in \mathbb{Z}_{+}^{m-1}, \partial_{l}=\partial_{2}^{l^{2}} \ldots \partial_{m}^{l^{m}}, u^{\sigma}=u_{00}^{\sigma 1}, u_{\kappa}^{\sigma \alpha}=u_{\kappa 0}^{\sigma \alpha}$. Согласно правилам алгебро-геометрического анализа пара $\mathcal{X}, \partial=\left(\partial_{1}, \ldots, \partial_{m}\right)$ описывает эволюционную систему

$$
\begin{gathered}
\partial_{x^{1}} u^{\sigma}=\mathrm{f}^{\sigma}=U_{10}^{\sigma 1}=-\sum_{2 \leqslant \beta \leqslant m} u_{0(\beta)}^{\sigma \beta}, \quad 1 \leqslant \sigma \leqslant s, \\
\partial_{x^{1}} u_{\kappa}^{\sigma \alpha}=\mathrm{f}_{\kappa}^{\sigma \alpha}=u_{\kappa+1}^{\sigma \alpha}, \quad 2 \leqslant \alpha \leqslant m, \quad \kappa \in \mathbb{Z}_{+},
\end{gathered}
$$

где $x^{1} \in \mathbb{R}$ - эволюционная переменная ("время"), ( $\left.x^{\alpha}\right) \in \mathbb{R}^{m-1}$ - пространственные переменные, $\left(u^{\sigma}, u_{\kappa}^{\sigma \alpha}\right) \in \mathbb{R}^{s} \times \mathbb{R}_{\mathbb{Z}_{+}^{s(m-1)}}$ - зависимые переменные, $\left(u_{l}^{\sigma}, u_{\kappa l}^{\sigma \alpha}\right) \in \mathbb{R}_{\mathbb{Z}_{+}^{m-1}}^{s} \times$ $\mathbb{R}_{\mathbb{Z}_{+}^{m}}^{s(m-1)}$ - дифференциальные переменные и дифференцирования $\partial_{\mu}$ (см. $(2.5),(2.6)$ ) суть частные производные в силу системы (2.7), (2.8), $1 \leqslant \mu \leqslant m$.

Таким образом, система уравнений неразрывности (2.1) эквивалентна эволюиионной системе (2.7), (2.8), и мы будем их отождествлять.

Удобство эволюционных систем в том, что для них имеется полное описание законов сохранения и симметрий (см., например, работы [2], [3], [6]). Ниже мы пользуемся техникой, развитой в работах [5], [7], [8].

2.4. Линейные операторы в полных производных. Введем $\mathcal{A}$-модуль $\mathcal{E}=\mathcal{A}^{s} \times \mathcal{A}_{\mathbb{Z}_{+}}^{s(m-1)}$ с элементами $\phi=\left(\phi^{\sigma}, \phi_{\kappa}^{\sigma \alpha}\right)$ и дуальный $\mathcal{A}$-модуль $\mathcal{E}^{*}=\mathcal{A}_{s} \times$ $\left(\mathcal{A}_{s(m-1)}^{\mathbb{Z}_{+}}\right)_{\text {fin }}$ с элементами $\chi=\left(\chi_{\sigma}, \chi_{\sigma \alpha}^{\kappa}\right)$, где лишь конечное число компонент $\chi_{\sigma \alpha}^{\kappa}$ ненулевые. Элемент $\mathrm{f}=\left(\mathrm{f}^{\sigma}, \mathrm{f}_{\kappa}^{\sigma \alpha}\right) \in \mathcal{E}$ с компонентами, задающими эволюционную систему (2.7), (2.8), порождает линейный оператор (универсальную линеаризацию)

$$
\mathrm{f}_{*}: \mathcal{E} \rightarrow \mathcal{E}, \quad \phi=\left(\phi^{\sigma}, \phi_{\kappa}^{\sigma \alpha}\right) \mapsto \mathrm{f}_{*}(\phi)=\left(\mathrm{f}_{*}(\phi)^{\sigma}, \mathrm{f}_{*}(\phi)_{\kappa}^{\sigma \alpha}\right),
$$


где

$$
\begin{aligned}
\mathrm{f}_{*}(\phi)^{\sigma} & =\sum_{\sigma^{\prime}, l} \partial_{u_{l}^{\sigma^{\prime}}}\left(\mathrm{f}^{\sigma}\right) \partial_{l} \phi^{\sigma^{\prime}}+\sum_{\sigma^{\prime}, \alpha^{\prime}, \kappa^{\prime}, l} \partial_{u_{\kappa^{\prime} l}^{\sigma^{\prime} \alpha^{\prime}}}\left(\mathrm{f}^{\sigma}\right) \partial_{l} \phi_{\kappa^{\prime}}^{\sigma^{\prime} \alpha^{\prime}}=-\sum_{\alpha} \partial_{\alpha} \phi^{\sigma \alpha}, \\
\mathrm{f}_{*}(\phi)_{\kappa}^{\sigma \alpha} & =\sum_{\sigma^{\prime}, l} \partial_{u_{l}^{\sigma^{\prime}}}\left(\mathrm{f}_{\kappa}^{\sigma \alpha}\right) \partial_{l} \phi^{\sigma^{\prime}}+\sum_{\sigma^{\prime}, \alpha^{\prime}, \kappa^{\prime}, l} \partial_{u_{\kappa^{\prime} l}^{\sigma^{\prime} \alpha^{\prime}}}\left(\mathrm{f}_{\kappa}^{\sigma \alpha}\right) \partial_{l} \phi_{\kappa^{\prime}}^{\sigma^{\prime} \alpha^{\prime}}=\phi_{\kappa+1}^{\sigma \alpha} ;
\end{aligned}
$$

напомним, что $\phi^{\sigma \alpha}=\phi_{0}^{\sigma \alpha}$. Дуальный (сопряженный по Лагранжу) оператор имеет вид

$$
\mathrm{f}^{*}: \mathcal{E}^{*} \rightarrow \mathcal{E}^{*}, \quad \chi=\left(\chi_{\sigma}, \chi_{\sigma \alpha}^{\kappa}\right) \mapsto \mathrm{f}^{*}(\chi)=\left(\mathrm{f}^{*}(\chi)_{\sigma}, \mathrm{f}^{*}(\chi)_{\sigma \alpha}^{\kappa}\right)
$$

где

$$
\begin{aligned}
\mathrm{f}^{*}(\chi)_{\sigma} & =\sum_{\sigma^{\prime}, l}(-1)^{|l|} \partial_{l}\left(\chi_{\sigma^{\prime}} \partial_{u_{l}^{\sigma}} \mathrm{f}^{\sigma^{\prime}}\right)+\sum_{\sigma^{\prime}, \alpha^{\prime}, \kappa^{\prime}, l}(-1)^{|l|} \partial_{l}\left(\chi_{\sigma^{\prime} \alpha^{\prime}}^{\kappa^{\prime}} \partial_{u_{l}^{\sigma}} \mathrm{f}_{\kappa^{\prime}}^{\sigma^{\prime} \alpha^{\prime}}\right)=0, \\
& = \begin{cases}\partial_{\alpha} \chi_{\sigma}, & \kappa=0, \\
\chi_{\sigma \alpha}^{\kappa-1}, & \kappa \in \mathbb{N} .\end{cases}
\end{aligned}
$$

Здесь $|l|=l^{2}+\cdots+l^{m}$.

2.5. Законы сохранения. Законы сохранения эволюционной системы полностью описываются своими характеристиками (см., например, работы [5], [7]). В нашем случае элемент $\chi \in \mathcal{E}^{*}$ является характеристикой закона сохранения системы (2.7), (2.8) тогда и только тогда, когда

$$
\begin{aligned}
\partial_{1} \chi+\mathrm{f}^{*}(\chi) & =0, \\
\chi_{*}-\chi^{*} & =0,
\end{aligned}
$$

где оператор $\chi_{*}: \mathcal{E} \rightarrow \mathcal{E}^{*}-$ универсальная линеаризация элемента $\chi$, а $\chi^{*}: \mathcal{E} \rightarrow \mathcal{E}^{*}-$ его дуальный. Операторное уравнение (2.12) по сути является системой уравнений для компонент $\chi_{\sigma}, \chi_{\sigma \alpha}^{\kappa}$ элемента $\chi$, называемой иногда условиями Гелъмголъца. Уравнения (2.11) подробнее записываются как

$$
\begin{aligned}
\partial_{1} \chi_{\sigma} & =0, \\
\partial_{1} \chi_{\sigma \alpha}^{0}+\partial_{\alpha} \chi_{\sigma} & =0, \\
\partial_{1} \chi_{\sigma \alpha}^{\kappa}+\chi_{\sigma \alpha}^{\kappa-1} & =0,
\end{aligned}
$$

где $1 \leqslant \sigma \leqslant s, 2 \leqslant \alpha \leqslant m, \kappa \in \mathbb{N}$. По определению элемент $\chi$ содержит лишь конечное число ненулевых компонент. Отсюда легко выводится, что подсистема (2.15) имеет единственное тривиальное решение $\chi_{\sigma \alpha}^{\kappa}=0$ для всех $\kappa \in \mathbb{Z}_{+}, 1 \leqslant \sigma \leqslant s$, $2 \leqslant \alpha \leqslant m$. Подсистема (2.14) тогда принимает вид

$$
\partial_{\alpha} \chi_{\sigma}=0, \quad 2 \leqslant \alpha \leqslant m, \quad 1 \leqslant \sigma \leqslant s,
$$


откуда в силу конечности порядка функций из $\mathcal{A}$ следует, что компоненты $\chi_{\sigma}=$ $\chi_{\sigma}\left(x^{1}\right)$ для всех $1 \leqslant \sigma \leqslant s$. Подсистема (2.13) теперь сводится к системе

$$
\partial_{x^{1}} \chi_{\sigma}=0, \quad 1 \leqslant \sigma \leqslant s,
$$

с общим решением $\chi_{\sigma}=$ const. В этом случае $\chi_{*}=\chi^{*}=0$, так что условия Гельмгольца (2.12) тривиально выполняются. Итак, доказана

Теорема 1. Общее решение системы (2.11), (2.12) имеет вид

$$
\chi=\sum_{\sigma} a_{\sigma} \chi^{\sigma}, \quad a_{\sigma} \in \mathbb{R},
$$

где базисные решения $\chi^{\sigma}=\left(e^{\sigma}, 0\right), e^{\sigma}=(0, \ldots, 1, \ldots, 0)$ (единица стоит на $\sigma$-м месте), $1 \leqslant \sigma \leqslant s$.

Напомним, что элемент $J=\left(j^{\mu} \mid 1 \leqslant \mu \leqslant m\right) \in \mathcal{A}^{m}$ называется сохраняющимся током системы (2.7), (2.8), если он удовлетворяет уравнению неразрывности

$$
\sum_{1 \leqslant \mu \leqslant m} \partial_{\mu} j^{\mu}=\operatorname{Div} J=0
$$

Уравнение неразрывности имеет очевидные решения (тривиальные токи) $J=\partial K=$ $\left((\partial K)^{\mu}\right)$ с компонентами $(\partial K)^{\mu}=\sum_{\nu} \partial_{\nu} k^{\mu \nu}$ с произвольными $k^{\mu \nu} \in \mathcal{A}, k^{\nu \mu}+k^{\mu \nu}=0$. Законы сохранения системы (2.7), (2.8) суть классы эквивалентности сохраняющихся токов по тривиальным токам. Согласно общей теории эволюционных систем (см., например, работы [5], [7]) линейное пространство законов сохранения системы (2.7), (2.8) изоморфно пространству их характеристик, т. е. решений системы (2.11), (2.12), где изоморфизм задается правилом

$$
[J]=\left[\left(j^{1}, \ldots, j^{m}\right)\right] \mapsto \chi=\left(\chi_{\sigma}, \chi_{\sigma \alpha}^{\kappa}\right), \quad \chi_{\sigma}=\frac{\delta j^{1}}{\delta u^{\sigma}}, \quad \chi_{\sigma \alpha}^{\kappa}=\frac{\delta j^{1}}{\delta u_{\kappa}^{\sigma \alpha}},
$$

квадратные скобки означают класс эквивалентности. В частности, доказанная выше теорема имеет

СлЕДСТВиЕ 1. Общее решение уравнения неразрывности $\operatorname{Div} J=0$ для системы (2.7), (2.8) имеет вид

$$
J=\sum_{1 \leqslant \sigma \leqslant s} a_{\sigma} J^{\sigma}+\partial K
$$

где базисные токи $J^{\sigma}=\left(u^{\sigma \mu}\right)$, а тривиальный ток $\partial K=\left(\sum_{\nu} \partial_{\nu} k^{\mu \nu}\right)$.

СЛЕДСТвИЕ 2. Линейное пространство $\mathrm{CL}(\mathcal{X})$ законов сохранения системы (2.7), (2.8), а значит и исходных уравнений неразрывности (2.1), имеет представления

$$
\begin{aligned}
\mathrm{CL}(\mathcal{X}) & =\left\{[J]=\sum_{1 \leqslant \sigma \leqslant s} a_{\sigma}\left[J^{\sigma}\right] \mid a_{\sigma} \in \mathbb{R}\right\} \simeq \\
& \simeq\left\{J=\sum_{1 \leqslant \sigma \leqslant s} a_{\sigma} J^{\sigma} \mid a_{\sigma} \in \mathbb{R}\right\} \simeq \mathbb{R}_{s} .
\end{aligned}
$$


2.6. Законы сохранения разных степеней. Для каждого $q \in \mathbb{Z}_{+}$определен $\mathcal{A}$-модуль

$$
\Omega_{\mathrm{H}}^{q}(\mathcal{X})=\left\{\omega=\frac{1}{q !} \sum_{1 \leqslant \mu_{1}, \ldots, \mu_{q} \leqslant m} \omega_{\mu_{1} \ldots \mu_{q}} \cdot d x^{\mu_{1}} \wedge \cdots \wedge d x^{\mu_{q}}\right\}
$$

горизонтальных q-борм на поверхности $\mathcal{X}$, где кососимметрические по индексам компоненты $\omega_{\mu_{1} \ldots \mu_{q}} \in \mathcal{A}$. Очевидно, что $\Omega_{\mathrm{H}}^{q}(\mathcal{X})=0$ при $q>m$. Будем также считать, что $\Omega_{\mathrm{H}}^{q}(\mathcal{X})=0$ при $q<0$. Горизонтальные внешние дифберенииаль запишем как

$$
d_{\mathrm{H}}=d_{\mathrm{H}}^{q}: \Omega_{\mathrm{H}}^{q}(\mathcal{X}) \rightarrow \Omega_{\mathrm{H}}^{q+1}(\mathcal{X}), \quad \omega \rightarrow d_{\mathrm{H}} \omega
$$

где

$$
\begin{gathered}
d_{\mathrm{H}} \omega=\frac{1}{(q+1) !} \sum_{1 \leqslant \mu_{0}, \ldots, \mu_{q} \leqslant m}\left(d_{\mathrm{H}} \omega\right)_{\mu_{0} \ldots \mu_{q}} d x^{\mu_{0}} \wedge \cdots \wedge d x^{\mu_{q}}, \\
\left(d_{\mathrm{H} \omega} \omega\right)_{\mu_{0} \ldots \mu_{q}}=\sum_{0 \leqslant r \leqslant q}(-1)^{r} \partial_{\mu_{r}} \omega_{\mu_{0} \ldots \widetilde{\mu_{r}} \ldots \mu_{q}},
\end{gathered}
$$

значок “ $\sim$ указывает, что эта переменная пропущена. Легко проверяется, что $d_{\mathrm{H}} \circ d_{\mathrm{H}}=0$.

Линейное пространство сохраняющихся токов q-й степени системы (2.7), (2.8)-

$$
\mathrm{CC}^{q}(\mathcal{X})=\operatorname{Ker} d_{\mathrm{H}}^{q}=\left\{\omega \in \Omega_{\mathrm{H}}^{q}(\mathcal{X}) \mid d_{\mathrm{H}} \omega=0\right\},
$$

а линейное пространство тривиальных токов q-й степени системы (2.7), (2.8) есть

$$
\operatorname{TC}^{q}(\mathcal{X})=\operatorname{Im} d_{\mathrm{H}}^{q-1}=\left\{\omega=d_{\mathrm{H}} \chi \mid \chi \in \Omega_{\mathrm{H}}^{q-1}(\mathcal{X})\right\} .
$$

Очевидно, что $\operatorname{TC}^{q}(\mathcal{X}) \subset \mathrm{CC}^{q}(\mathcal{X})$, так что определено фактор-пространство

$$
\mathrm{CL}^{q}(\mathcal{X})=\mathrm{CC}^{q}(\mathcal{X}) / \mathrm{TC}^{q}(\mathcal{X})=\operatorname{Ker} d_{\mathrm{H}}^{q} / \operatorname{Im} d_{\mathrm{H}}^{q-1}=H_{\mathrm{H}}^{q}(\mathcal{X})
$$

- линейное пространство законов сохранения q-й степени системы (2.7), (2.8).

Как у всякой эволюционной системы (см., например, работы [5], [9], [10]), у системы $(2.7),(2.8)$ :

- $\mathrm{CL}^{0}(\mathcal{X})=\mathbb{R}$

- $\mathrm{CL}^{q}(\mathcal{X})=0$ при $0<q<m-1$;

- $\mathrm{CL}^{m-1}(\mathcal{X})=\mathrm{CL}(\mathcal{X})$ - пространство обычных законов сохранения, вычисленное выше (см. следствие 2); соответствие между определениями этого и предыдущего пунктов дается правилами

$$
\begin{aligned}
& J=\left(j^{\mu}\right) \mapsto \omega=\sum_{\mu} j^{\mu} d_{\mu} x, \quad d_{\mu} x=(-1)^{\mu-1} d x^{1} \wedge \cdots \wedge \overline{d x^{\mu}} \wedge \cdots \wedge d x^{m} ; \\
& K=\left(k^{\mu \nu}\right) \mapsto \chi=\sum_{\mu<\nu} k^{\mu \nu} d_{\mu \nu} x, \quad d_{\mu \nu} x=(-1)^{\mu+\nu} d x^{1} \wedge \cdots \wedge \overline{d x^{\mu}} \ldots \overline{d x^{\nu}} \wedge \cdots \wedge d x^{m}
\end{aligned}
$$

- $\mathrm{CL}^{m}(\mathcal{X})=\Omega_{\mathrm{H}}^{m}(\mathcal{X}) / \operatorname{Im} d_{\mathrm{H}}^{m-1}-$ пространство функционалов на поверхности $\mathcal{X}$. 
2.7. Симметрии. Симметрии (инфинитезимальные симметрии) системы (2.7), (2.8) суть вертикальные дифференцирования

$$
V=\sum_{\sigma, l} V_{l}^{\sigma} \partial_{u_{l}^{\sigma}}+\sum_{\sigma, \alpha, \kappa, l} V_{\kappa l}^{\sigma \alpha} \partial_{u_{\kappa l}^{\sigma \alpha}},
$$

для которых коммутаторы $\left[V, \partial_{\mu}\right]=0,1 \leqslant \mu \leqslant m$. Для этого необходимо и достаточно, чтобы компоненты дифференцирования $V$ имели вид $V_{l}^{\sigma}=\partial_{l} \phi^{\sigma}, V_{\kappa l}^{\sigma \alpha}=\partial_{l} \phi_{\kappa}^{\sigma \alpha}$, где элемент $\phi=\left(\phi^{\sigma}, \phi_{\kappa}^{\sigma \alpha}\right) \in \mathcal{E}$ удовлетворяет уравнению

$$
\partial_{1} \phi-\mathrm{f}_{*}(\phi)=0
$$

Подробнее,

$$
\begin{aligned}
& \sum_{1 \leqslant \mu \leqslant m} \partial_{\mu} \phi^{\sigma \mu}=0 \\
& \partial_{1} \phi_{\kappa}^{\sigma \alpha}-\phi_{\kappa+1}^{\sigma \alpha}=0
\end{aligned}
$$

для всех $1 \leqslant \sigma \leqslant s, 2 \leqslant \alpha \leqslant m, \kappa \in \mathbb{Z}_{+}$, где $\phi^{\sigma 1}=\phi^{\sigma}, \phi^{\sigma \alpha}=\phi_{0}^{\sigma \alpha}$. В силу следствия 1 общее решение подсистемы (2.17) имеет вид

$$
\phi^{\sigma \mu}=\sum_{\sigma^{\prime}} a_{\sigma^{\prime}}^{\sigma} u^{\sigma^{\prime} \mu}+\sum_{\nu} \partial_{\nu} k^{\sigma \mu \nu}, \quad a_{\sigma^{\prime}}^{\sigma} \in \mathbb{R}, \quad k^{\sigma \mu \nu} \in \mathcal{A}, \quad k^{\sigma \mu \nu}+k^{\sigma \nu \mu}=0,
$$

для всех $1 \leqslant \sigma \leqslant s, 1 \leqslant \mu, \nu \leqslant m$. В свою очередь подсистема (2.18) дает

$$
\phi_{\kappa}^{\sigma \alpha}=\partial_{1}^{\kappa} \phi^{\sigma \alpha}, \quad 1 \leqslant \sigma \leqslant s, \quad 2 \leqslant \alpha \leqslant m, \quad \kappa \in \mathbb{Z}_{+} .
$$

Таким образом, справедлива

Теорема 2. Симметрии системы (2.7), (2.8), а значит и исходных уравнений неразрывности (2.1), суть дифферениирования вида (2.16), где компоненть $\phi^{\sigma \mu}$ имеют вид (2.19), $\phi^{\sigma}=\phi^{\sigma 1}$, а компоненты $\phi_{\kappa}^{\sigma \alpha}$ находятся по формулам (2.20).

Другими словами, линейное пространство $\operatorname{Sym}(\mathcal{X})$ симметрий системь (2.1) имеет представления

$$
\begin{aligned}
\operatorname{Sym}(\mathcal{X}) & \simeq\left\{\Phi^{\sigma}=\sum_{\sigma^{\prime}} a_{\sigma^{\prime}}^{\sigma} J^{\sigma^{\prime}}+\partial K^{\sigma} \mid a_{\sigma^{\prime}}^{\sigma} \in \mathbb{R}, 1 \leqslant \sigma, \sigma^{\prime} \leqslant s\right\} \simeq \\
& \simeq \mathrm{CC}^{m-1}(\mathcal{X})^{s},
\end{aligned}
$$

где $\Phi^{\sigma}=\left(\phi^{\sigma \mu}\right), J^{\sigma}=\left(u^{\sigma \mu}\right), \partial K^{\sigma}=\left(\sum_{\nu} \partial_{\nu} k^{\sigma \mu \nu}\right)$.

\section{3. АЛГЕБРО-ГЕОМЕТРИЧЕСКИЙ АНАЛИЗ УРАВНЕНИЙ, ОПИСЫВАЮЩИХ ЭВОЛЮЦИЮ С НУЛЕВОЙ ДИВЕРГЕНЦИЕЙ}

3.1. Обозначения. K введенным выше обозначениям добавим новые:

- $t \in \mathbb{R}$ - независимая переменная (время);

- $\partial_{t}$ - частная производная по переменной $t$; 
- $\mathfrak{B}=\mathcal{C}^{\infty}(\mathbb{R}) \otimes \mathfrak{A}$ - алгебра дифференциальных функций конечного порядка на пространстве $\mathbb{R}^{1+m} \times \mathbb{R}_{\mathbb{Z}_{+}^{m}}^{s m}$;

- $\mathcal{B}=\mathcal{C}^{\infty}(\mathbb{R}) \otimes \mathcal{A}$ - алгебра дифференциальных функций конечного порядка на пространстве $\mathbb{R}^{1+m} \times \mathbb{R}_{\mathbb{Z}_{+}^{m-1}}^{s} \times \mathbb{R}_{\mathbb{Z}_{+}^{m}}^{s(m-1)}$.

Имеются естественные вложения $\mathfrak{A} \subset \mathfrak{B}$ и $\mathcal{A} \subset \mathcal{B}$, задаваемые правилом $f \mapsto$ $1(t) \otimes f$, где функция $1(t) \equiv 1$ для всех $t \in \mathbb{R}$. Очевидно, все построения предыдущего раздела сохранят свою силу, если считать, что введенные там функции зависят от переменной $t$ как от параметра.

3.2. Эволюция с нулевой дивергенцией. Здесь эволюция переменных $u=$ $\left(u^{\sigma \mu}\right) \in \mathbb{R}^{s m}$ в пространстве $\mathbb{R}^{m}$ описывается системой

$$
\partial_{t} u^{\sigma \mu}=F^{\sigma \mu}, \quad 1 \leqslant \sigma \leqslant s, \quad 1 \leqslant \mu \leqslant m,
$$

при наличии связей

$$
\sum_{1 \leqslant \mu \leqslant m} \partial_{x^{\mu}} u^{\sigma \mu}=0, \quad 1 \leqslant \sigma \leqslant s,
$$

где $F^{\sigma \mu}=F^{\sigma \mu}(t, x, \mathbf{u}) \in \mathfrak{B}$ - заданные функции.

Согласно упомянутым выше правилам алгебро-геометрического анализа (см., например, работы [1]-[6]) система уравнений (3.1), (3.2) определяет гладкую поверхность $\mathcal{T} \mathcal{X}=\mathbb{R} \times \mathcal{X} \subset \mathbb{R}^{1+m} \times \mathbb{R}_{\mathbb{Z}_{+}^{m}}^{s m}$. Глобальные координаты на поверхности $\mathcal{T} \mathcal{X}$ суть переменные $t \in \mathbb{R}, x=\left(x^{\mu}\right) \in \mathbb{R}^{m}$ и $\left(u_{l}^{\sigma}, u_{\kappa l}^{\sigma \alpha}\right) \in \mathbb{R}_{\mathbb{Z}_{+}^{m-1}}^{s} \times \mathbb{R}_{\mathbb{Z}_{+}^{m}}^{s(m-1)}$. К картановым дифференцированиям $\partial_{\mu}, 1 \leqslant \mu \leqslant m$ (см. (2.5), (2.6)), добавляется дифференцирование

$$
\partial_{0}=\partial_{t}+V, \quad V=\sum_{\sigma, l} V_{l}^{\sigma} \partial_{u_{l}^{\sigma}}+\sum_{\sigma, \alpha, \kappa, l} V_{\kappa l}^{\sigma \alpha} \partial_{u_{\kappa l}^{\sigma \alpha}} .
$$

Эволюция (3.1) согласована со связями (3.2), если дифференцирование (3.3) эволющионное, т.е. если вертикальное дифференцирование $V$ есть симметрия системы (3.2). Согласно теореме 2 для этого необходимо и достаточно, чтобы компоненты $V_{l}^{\sigma}=\partial_{l} \phi^{\sigma 1}, V_{\kappa l}^{\sigma \alpha}=\partial_{1}^{\kappa} \partial_{l} \phi^{\sigma \alpha}$, где

$$
\phi^{\sigma \mu}=\left.F^{\sigma \mu}\right|_{\mathcal{T} \mathcal{X}}=\sum_{\sigma^{\prime}} a_{\sigma^{\prime}}^{\sigma} u^{\sigma^{\prime} \mu}+\sum_{\nu} \partial_{\nu} k^{\sigma \mu \nu},
$$

$a_{\sigma^{\prime}}^{\sigma}=a_{\sigma^{\prime}}^{\sigma}(t) \in \mathcal{C}^{\infty}(\mathbb{R}), k^{\sigma \mu \nu} \in \mathcal{B}, k^{\sigma \mu \nu}+k^{\sigma \nu \mu}=0,1 \leqslant \sigma, \sigma^{\prime} \leqslant s, 1 \leqslant \mu, \nu \leqslant m$.

Ниже мы будем считать, что эволющия согласована со связями.

3.3. Законы сохранения разных степеней. Для каждого $q \in \mathbb{Z}_{+}$определен $\mathcal{B}$-модуль

$$
\Omega_{\mathrm{X}}^{q}(\mathcal{T} \mathcal{X})=\left\{\omega=\frac{1}{q !} \sum_{1 \leqslant \mu_{1}, \ldots, \mu_{q} \leqslant m} \omega_{\mu_{1} \ldots \mu_{q}} \cdot d x^{\mu_{1}} \wedge \cdots \wedge d x^{\mu_{q}}\right\}
$$

пространственных q-форм на поверхности $\mathcal{T} \mathcal{X}$, где кососимметрические по индексам компоненты $\omega_{\mu_{1} \ldots \mu_{q}} \in \mathcal{B}$. Очевидно, что $\Omega_{\mathrm{X}}^{q}(\mathcal{T} \mathcal{X})=0$ при $q>m$. Будем также считать, что $\Omega_{\mathrm{X}}^{q}(\mathcal{T} \mathcal{X})=0$ при $q<0$. Пространственные внешние дифференииаль

$$
d_{\mathrm{X}}=d_{\mathrm{X}}^{q}: \Omega_{\mathrm{X}}^{q}(\mathcal{T} \mathcal{X}) \rightarrow \Omega_{\mathrm{X}}^{q+1}(\mathcal{T} \mathcal{X}), \quad \omega \rightarrow d_{\mathrm{X}} \omega,
$$


где

$$
\begin{gathered}
d_{\mathrm{X}} \omega=\frac{1}{(q+1) !} \sum_{1 \leqslant \mu_{0}, \ldots, \mu_{q} \leqslant m}\left(d_{\mathrm{X}} \omega\right)_{\mu_{0} \ldots \mu_{q}} \cdot d x^{\mu_{0}} \wedge \cdots \wedge d x^{\mu_{q}}, \\
\left(d_{\mathrm{X}} \omega\right)_{\mu_{0} \ldots \mu_{q}}=\sum_{0 \leqslant r \leqslant q}(-1)^{r} \partial_{\mu_{r}} \omega_{\mu_{0} \ldots \widetilde{\mu_{r}}} \ldots \mu_{q} .
\end{gathered}
$$

Легко проверяется, что $d_{\mathrm{X}} \circ d_{\mathrm{X}}=0$. При вычислении пространственного дифференциала $d_{\mathrm{X}}$ переменная $t \in \mathbb{R}$ является параметром, так что вложение $\mathcal{A} \subset \mathcal{B}$ приводит к вложениям $\Omega_{\mathrm{H}}^{q}(\mathcal{X}) \subset \Omega_{\mathrm{X}}^{q}(\mathcal{T} \mathcal{X})$, причем $\left.d_{\mathrm{X}}^{q}\right|_{\Omega_{\mathrm{H}}^{q}(\mathcal{X})}=d_{\mathrm{H}}^{q}$. Отсюда легко выводится представление

$$
H_{\mathrm{X}}^{q}(\mathcal{T} \mathcal{X})=\operatorname{Ker} d_{\mathrm{X}}^{q} / \operatorname{Im} d_{\mathrm{X}}^{q-1}=\mathcal{C}^{\infty}(\mathbb{R}) \otimes \operatorname{CL}^{q}(\mathcal{X}), \quad 0 \leqslant q \leqslant m,
$$

так что

$$
\begin{gathered}
H_{\mathrm{X}}^{0}(\mathcal{T} \mathcal{X})=\mathcal{C}^{\infty}(\mathbb{R}), \\
H_{\mathrm{X}}^{q}(\mathcal{T} \mathcal{X})=0, \quad 1 \leqslant q \leqslant m-2, \\
H_{\mathrm{X}}^{m-1}(\mathcal{T} \mathcal{X})=\left\{J=\sum_{1 \leqslant \sigma \leqslant s} a_{\sigma} J^{\sigma} \mid a_{\sigma}=a_{\sigma}(t) \in \mathcal{C}^{\infty}(\mathbb{R})\right\}, \\
H_{\mathrm{X}}^{m}(\mathcal{T} \mathcal{X})=\Omega_{\mathrm{X}}^{m}(\mathcal{T} \mathcal{X}) / \operatorname{Im} d_{\mathrm{X}}^{m-1} .
\end{gathered}
$$

Для каждого $q \in \mathbb{Z}_{+}$определен $\mathcal{B}$-модуль

$$
\begin{aligned}
\Omega_{\mathrm{H}}^{q}(\mathcal{T} \mathcal{X}) & =\left\{\omega=\phi+d t \wedge \psi \mid \phi \in \Omega_{\mathrm{X}}^{q}(\mathcal{T} \mathcal{X}), \psi \in \Omega_{\mathrm{X}}^{q-1}(\mathcal{T} \mathcal{X})\right\}= \\
& =\Omega_{\mathrm{X}}^{q}(\mathcal{T} \mathcal{X}) \oplus d t \wedge \Omega_{\mathrm{X}}^{q-1}(\mathcal{T} \mathcal{X})
\end{aligned}
$$

горизонтальных q-форм на поверхности $\mathcal{T} \mathcal{X}$. Эволюционные внешние дифференииальи имеют вид

$$
d_{\mathrm{T}}=d_{\mathrm{T}}^{q}: \Omega_{\mathrm{H}}^{q}(\mathcal{T} \mathcal{X}) \rightarrow \Omega_{\mathrm{H}}^{q+1}(\mathcal{T} \mathcal{X}), \quad \omega \mapsto d_{\mathrm{T}} \omega=d t \wedge \partial_{0} \omega,
$$

где дифференцирование $\partial_{0}$ действует на форму $\omega$ как производная Ли, здесь переменная $t$ - полноправный аргумент. Очевидно, $d_{\mathrm{T}} \circ d_{\mathrm{T}}=0$. Горизонтальные внешние дифференциаль запишем как

$$
\begin{gathered}
d_{\mathrm{H}}=d_{\mathrm{H}}^{q}=d_{\mathrm{T}}^{q}+d_{\mathrm{X}}^{q}: \Omega_{\mathrm{H}}^{q}(\mathcal{T} \mathcal{X}) \rightarrow \Omega_{\mathrm{H}}^{q+1}(\mathcal{T} \mathcal{X}), \\
\omega=\phi+d t \wedge \psi \mapsto d_{\mathrm{H}} \omega=d_{\mathrm{X}} \phi+d t \wedge\left(\partial_{0} \phi-d_{\mathrm{X}} \psi\right) .
\end{gathered}
$$

Легко проверяется, что $d_{\mathrm{T}} \circ d_{\mathrm{X}}+d_{\mathrm{X}} \circ d_{\mathrm{T}}=0$, так что $d_{\mathrm{H}} \circ d_{\mathrm{H}}=0$.

Линейное пространство сохраняющихся токов q-й степени системы (3.1), (3.2) есть $\operatorname{CC}^{q}(\mathcal{T} \mathcal{X})=\operatorname{Ker} d_{\mathrm{H}}^{q}$. Линейное пространство тривиальных токов $q$-й степени системы $(3.1),(3.2)$ есть $\operatorname{TC}^{q}(\mathcal{T} \mathcal{X})=\operatorname{Im} d_{\mathrm{H}}^{q-1}$. Очевидно, что $\operatorname{TC}^{q}(\mathcal{T} \mathcal{X}) \subset \mathrm{CC}^{q}(\mathcal{T} \mathcal{X})$, так что определено фактор-пространство $\mathrm{CL}^{q}(\mathcal{T} \mathcal{X})=\mathrm{CC}^{q}(\mathcal{T} \mathcal{X}) / \mathrm{TC}^{q}(\mathcal{T} \mathcal{X})$ - линейное пространство законов сохранения q-й степени системы (3.1), (3.2). 
3.4. Переплетающая последовательность. Для изучения законов сохранения системы (3.1), (3.2) воспользуемся переплетающей последовательностью (точной последовательностью линейных пространств), построенной в работе [8]. В нашем случае она имеет вид

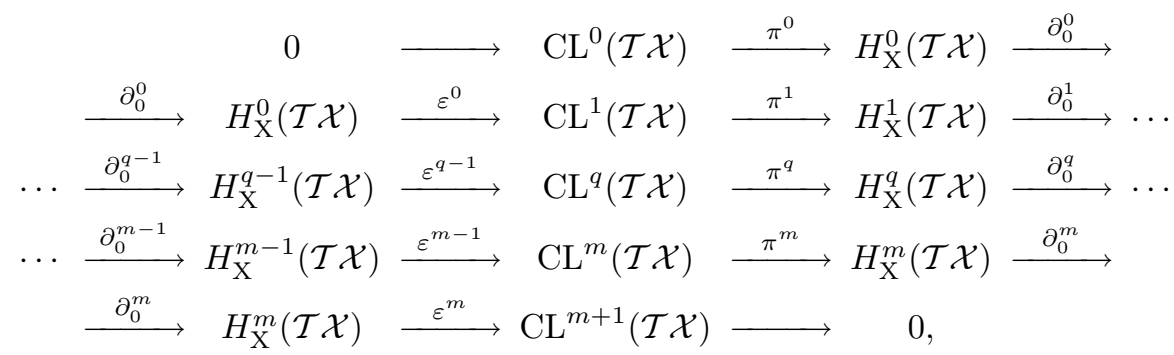

где

$$
\begin{aligned}
& \varepsilon^{q}: H_{\mathrm{X}}^{q}(\mathcal{T} \mathcal{X}) \rightarrow \mathrm{CL}^{q+1}(\mathcal{T} \mathcal{X}), \quad[\phi] \mapsto(-1)^{q}[d t \wedge \phi], \\
& \pi^{q}: \mathrm{CL}^{q}(\mathcal{T} \mathcal{X}) \rightarrow H_{\mathrm{X}}^{q}(\mathcal{T} \mathcal{X}), \quad[\phi+d t \wedge \psi] \mapsto[\phi], \\
& \partial_{0}^{q}: H_{\mathrm{X}}^{q}(\mathcal{T} \mathcal{X}) \rightarrow H_{\mathrm{X}}^{q}(\mathcal{T} \mathcal{X}), \quad[\phi] \mapsto\left[\partial_{0} \phi\right],
\end{aligned}
$$

- линейные отображения, квадратные скобки означают класс эквивалентности данной формы в соответствующем фактор-пространстве. В силу равенств (3.7) переплетающая последовательность распадается на точные подпоследовательности

$$
\begin{aligned}
& 0 \longrightarrow \mathrm{CL}^{0}(\mathcal{T} \mathcal{X}) \stackrel{\pi^{0}}{\longrightarrow} H_{\mathrm{X}}^{0}(\mathcal{T} \mathcal{X}) \stackrel{\partial_{0}^{0}}{\longrightarrow} H_{\mathrm{X}}^{0}(\mathcal{T} \mathcal{X}) \longrightarrow 0, \\
& 0 \longrightarrow \mathrm{CL}^{q}(\mathcal{T} \mathcal{X}) \longrightarrow 0, \quad 1 \leqslant q \leqslant m-2, \\
& 0 \longrightarrow \mathrm{CL}^{m-1}(\mathcal{T} \mathcal{X}) \stackrel{\pi^{m-1}}{\longrightarrow} H_{\mathrm{X}}^{m-1}(\mathcal{T} \mathcal{X}) \stackrel{\partial_{0}^{m-1}}{\longrightarrow} H_{\mathrm{X}}^{m-1}(\mathcal{T} \mathcal{X}), \\
& H_{\mathrm{X}}^{m-1}(\mathcal{T} \mathcal{X}) \stackrel{\varepsilon^{m-1}}{\longrightarrow} \mathrm{CL}^{m}(\mathcal{T} \mathcal{X}) \stackrel{\pi^{m}}{\longrightarrow} H_{\mathrm{X}}^{m}(\mathcal{T} \mathcal{X}) \stackrel{\partial_{0}^{m}}{\longrightarrow} H_{\mathrm{X}}^{m}(\mathcal{T} \mathcal{X}), \\
& H_{\mathrm{X}}^{m}(\mathcal{T} \mathcal{X}) \stackrel{\partial_{0}^{m}}{\longrightarrow} H_{\mathrm{X}}^{m}(\mathcal{T} \mathcal{X}) \stackrel{\varepsilon^{m}}{\longrightarrow} \mathrm{CL}^{m+1}(\mathcal{T} \mathcal{X}) \longrightarrow 0 .
\end{aligned}
$$

Отсюда с помощью простых вычислений выводится

ТЕОРема 3. Законы сохранения системы (3.1), (3.2) имеют представления

$$
\begin{gathered}
\mathrm{CL}^{0}(\mathcal{T} \mathcal{X})=\operatorname{Ker} \partial_{0}^{0}=\mathbb{R}, \\
\operatorname{CL}^{q}(\mathcal{T} \mathcal{X})=0, \quad 1 \leqslant q \leqslant m-2, \\
\pi^{m-1}: \mathrm{CL}^{m-1}(\mathcal{T} \mathcal{X}) \simeq \operatorname{Ker} \partial_{0}^{m-1}, \\
\Pi^{m}: \mathrm{CL}^{m}(\mathcal{T} \mathcal{X}) / \operatorname{Im} \varepsilon^{m-1} \simeq \operatorname{Ker} \partial_{0}^{m}, \\
E^{m}: \operatorname{coKer} \partial_{0}^{m}=H_{\mathrm{X}}^{m}(\mathcal{T} \mathcal{X}) / \operatorname{Im} \partial_{0}^{m} \simeq \mathrm{CL}^{m+1}(\mathcal{T} \mathcal{X}),
\end{gathered}
$$

где изоморфизм $\Pi^{m}$ действует по правилу

$$
[\phi+d t \wedge \psi]=\phi+d t \wedge \psi+\operatorname{Im} d_{\mathrm{H}}^{m-1}+\operatorname{Im} \varepsilon^{m-1} \mapsto[\phi]=\phi+\operatorname{Im} d_{\mathrm{X}}^{m-1}
$$

(напомним, что здесъ $d_{\mathrm{X}} \phi=0, \partial_{0} \phi=d_{\mathrm{X}} \psi$ ), а изоморфизм $E^{m}$ - по правилу

$$
[\phi]=\phi+\operatorname{Im} d_{\mathrm{X}}^{m-1}+\operatorname{Im} \partial_{0}^{m} \mapsto[d t \wedge \phi]=d t \wedge \phi+\operatorname{Im} d_{\mathrm{H}}^{m} .
$$

Здесъ $\phi \in \Omega_{\mathrm{X}}^{m}(\mathcal{T} \mathcal{X})$. 
ЗАмечАниЕ 1. По данному классу эквивалентности $[\phi] \in \operatorname{Ker} \partial_{0}^{m-1}$ класс эквивалентности $[\omega] \in \mathrm{CL}^{m-1}(\mathcal{T} \mathcal{X})$ находится следующим образом. По условию $d_{\mathrm{X}} \phi=0$, $\partial_{0} \phi=d_{\mathrm{X}} \psi$ для некоторой формы $\psi \in \Omega_{\mathrm{X}}^{m-2}(\mathcal{T} \mathcal{X})$. Положим $\omega=\phi+d t \wedge \psi$, тогда по построению $\pi^{m-1}[\omega]=[\phi]$, причем $d_{\mathrm{H}} \omega=d_{\mathrm{X}} \phi+d t \wedge\left(\partial_{0} \phi-d_{\mathrm{X}} \psi\right)=0$, что и требуется.

3.5. Законы сохранения $(m-1)$-й степени. Наибольший интерес в доказанной выше теореме представляет равенство (3.17), из которого следует, что связи (3.2) могут привести к появлению у эволюционной системы (3.1) законов сохранения $(m-1)$-й степени (напомним, что у эволюционных систем без связей возможны законы сохранения лишь $m$-й степени, где $m$ - число пространственных переменных). Для этого надо, чтобы ядро Ker $\partial_{0}^{m-1}$ отображения $\partial_{0}^{m-1}: H_{\mathrm{X}}^{m-1}(\mathcal{T} \mathcal{X}) \rightarrow H_{\mathrm{X}}^{m-1}(\mathcal{T} \mathcal{X})$ было нетривиальным. В нашем случае дифференцирование $\partial_{0}$ определено равенством (3.3), где компоненты $\phi^{\sigma \mu}$ имеют вид (3.4). Для базисного тока $J^{\sigma}=\left(j^{\sigma \mu}\right)$, $1 \leqslant \sigma \leqslant s$, действие $\partial_{0} J^{\sigma}=\left(\partial_{0} j^{\sigma \mu}\right)$, где (напомним, что $\left.j^{\sigma \mu}=u^{\sigma \mu}\right)$

$$
\partial_{0} j^{\sigma \mu}=\partial_{0} u^{\sigma \mu}=\phi^{\sigma \mu}=\sum_{\sigma^{\prime}} a_{\sigma^{\prime}}^{\sigma} j^{\sigma^{\prime} \mu}+\sum_{\nu} \partial_{\nu} k^{\sigma \mu \nu} \simeq \sum_{\sigma^{\prime}} a_{\sigma^{\prime}}^{\sigma} j^{\sigma^{\prime} \mu}
$$

(тривиальную часть $\sum_{\nu} \partial_{\nu} k^{\sigma \mu \nu}$ без ограничения общности отбрасываем). В матричных обозначениях это представление переписывается в виде $\partial_{0} \mathbb{J}=A \mathbb{J}$, где $s$-столбец $\mathbb{J}=\left(J^{\sigma}\right),(s \times s)$-матрица $A=\left\|a_{\sigma^{\prime}}^{\sigma}\right\|$. Следовательно, для произвольного нетривиального тока $J=\sum_{\sigma} c_{\sigma} J^{\sigma}$ с компонентами $c_{\sigma}=c_{\sigma}(t) \in \mathcal{C}^{\infty}(\mathbb{R})$ будем иметь

$$
\partial_{0} J=\sum_{\sigma}\left(\left(\partial_{t} c_{\sigma}\right) J^{\sigma}+c_{\sigma}\left(\partial_{0} J^{\sigma}\right)\right)=\left(\partial_{t} c+c A\right) \mathbb{J},
$$

где $s$-строка $c=\left(c_{\sigma}\right)$. Таким образом, справедлива

Лемма 1. Имеют место представления

$$
\begin{aligned}
\operatorname{Ker} \partial_{0}^{m-1} & =\left\{[\omega] \in H_{\mathrm{X}}^{m-1}(\mathcal{T} \mathcal{X}) \mid\left[\partial_{0} \omega\right]=0\right\}=\left\{J=\sum_{\sigma} c_{\sigma} J^{\sigma} \mid \partial_{0} J=0\right\}= \\
& =\left\{J=c \mathbb{J} \mid \partial_{t} c+c A=0\right\}=\left\{c \in \mathcal{C}^{\infty}(\mathbb{R})_{s} \mid \partial_{t} c+c A=0\right\} \simeq \mathbb{R}_{s} .
\end{aligned}
$$

Для доказательства следует лишь пояснить, что последний изоморфизм задается правилом $c(t) \mapsto c(0)$, и по сути данная лемма есть переформулировка теоремы о существовании и единственности решения задачи Коши для системы линейных обыкновенных дифференциальных уравнений первого порядка.

ЗАмЕчАниЕ 2. Если сужение $\phi^{\sigma \mu}=\sum_{\nu} \partial_{\nu} K^{\sigma \mu \nu}$ (см. представление (3.4)) содержит только тривиальную часть, то матрица $A=0$, так что ядро $\operatorname{Ker} \partial_{0}^{m-1}=\{J=$ $\left.c \mathbb{J} \mid c \in \mathbb{R}_{s}\right\}$.

Теорема 4. Имеют место представления

$$
\mathrm{CL}^{m-1}(\mathcal{T} \mathcal{X}) \simeq\left\{J=c \mathbb{J} \mid \partial_{t} c+c A=0\right\} \simeq\left\{c \in \mathcal{C}^{\infty}(\mathbb{R})_{s} \mid \partial_{t} c+c A=0\right\} \simeq \mathbb{R}_{s}
$$


Для доказательства достаточно воспользоваться равенством (3.17) и предыдущей леммой.

Таким образом, наличие связей в виде уравнений неразрывности действительно приводит к появлению у эволючионной системы законов сохранения низшей степени $m-1$.

3.6. Эволюция с заданной дивергенцией. Здесь эволюция переменных $v=$ $\left(v^{\sigma \mu}\right) \in \mathbb{R}^{s m}$ в пространстве $\mathbb{R}^{m}$ описывается системой

$$
\partial_{t} v^{\sigma \mu}=G^{\sigma \mu}, \quad 1 \leqslant \sigma \leqslant s, \quad 1 \leqslant \mu \leqslant m,
$$

при наличии связей

$$
\sum_{1 \leqslant \mu \leqslant m} \partial_{x^{\mu}} v^{\sigma \mu}=\varrho^{\sigma}, \quad 1 \leqslant \sigma \leqslant s,
$$

где $G^{\sigma \mu}=G^{\sigma \mu}(t, x, \mathbf{v}) \in \mathfrak{B}, \varrho^{\sigma}=\varrho^{\sigma}(t, x) \in \mathcal{C}^{\infty}\left(\mathbb{R}^{1+m}\right)$ - заданные функции. Такая эволюция сводится к рассмотренной выше эволюции с нулевой дивергенцией элементарной заменой

$$
\begin{aligned}
v^{\sigma \mu} & \longleftrightarrow u^{\sigma \mu}=v^{\sigma \mu}-R^{\sigma \mu} \\
G^{\sigma \mu}(t, x, \mathbf{v}) & \longleftrightarrow F^{\sigma \mu}(t, x, \mathbf{u})=G^{\sigma \mu}(t, x, \mathbf{v}-\mathbf{R})-\partial_{t} R^{\sigma \mu}, \\
\varrho^{\sigma} & \longleftrightarrow 0
\end{aligned}
$$

где $R^{\sigma \mu}=R^{\sigma \mu}(t, x)$ - компоненты произвольного частного решения уравнений неразрывности (3.21), $\mathbf{R}=\left(R_{i}^{\sigma \mu}\right), R_{i}^{\sigma \mu}=R_{i}^{\sigma \mu}(t, x)=\partial_{x^{i}} R^{\sigma \mu}(t, x)$.

3.7. Пример: уравнения Максвелла. Как известно, классическое электромагнитное поле описывается уравнениями Максвелла

$$
\begin{gathered}
\partial_{t} \mathbf{H}=-\operatorname{rot} \mathbf{E}, \\
\operatorname{div} \mathbf{H}=0,
\end{gathered}
$$

И

$$
\begin{gathered}
\partial_{t} \mathbf{E}=\operatorname{rot} \mathbf{H}-\mathbf{j}, \\
\operatorname{div} \mathbf{E}=\varrho,
\end{gathered}
$$

где $\mathbf{j}(t, x), \varrho(t, x)$ - заданные функции, удовлетворяющие уравнению неразрывности

$$
\partial_{t} \varrho+\operatorname{div} \mathbf{j}=0 .
$$

В силу уравнения (3.26) существуют функции $\mathbf{R}(t, x), \mathbf{S}(t, x)$ такие, что

$$
\varrho=\operatorname{div} \mathbf{R}, \quad \mathbf{j}=-\partial_{t} \mathbf{R}+\operatorname{rot} \mathbf{S},
$$

что позволяет переписать вторую пару уравнений Максвелла в более удобном для нас виде:

$$
\begin{gathered}
\partial_{t}(\mathbf{E}-\mathbf{R})=\operatorname{rot}(\mathbf{H}-\mathbf{S}), \\
\operatorname{div}(\mathbf{E}-\mathbf{R})=0 .
\end{gathered}
$$


С рассматриваемой здесь точки зрения уравнения (3.22), (3.28) описывают эволюцию переменных $\mathbf{H}, \mathbf{E}-\mathbf{R}$ с нулевой дивергенцией (3.23), (3.29). Таким образом, здесь $s=2, \sigma=1,2, m=3,1 \leqslant \lambda, \mu, \nu \leqslant 3, u^{1 \lambda}=H^{\lambda}, u^{2 \lambda}=E^{\lambda}-R^{\lambda}\left(E^{\lambda}=u^{2 \lambda}+R^{\lambda}\right)$,

$$
\begin{gathered}
F^{1 \lambda}=-\operatorname{rot} \mathbf{E}^{\lambda}=-\sum_{\mu, \nu} e^{\lambda \mu \nu} \partial_{\mu} E_{\nu} \\
F^{2 \lambda}=\operatorname{rot}(\mathbf{H}-\mathbf{S})^{\lambda}=\sum_{\mu, \nu} e^{\lambda \mu \nu} \partial_{\mu}\left(H_{\nu}-S_{\nu}\right),
\end{gathered}
$$

где $e^{\lambda \mu \nu}-$ знак перестановки $\{\lambda, \mu, \nu\}$, индексы $\lambda, \mu, \nu$ опускаются и поднимаются с помощью евклидовой метрики $\delta_{\mu \nu}, V_{\mu}=\sum_{\nu} \delta_{\mu \nu} V^{\nu}=V^{\mu}$. Далее, здесь сужение $\phi^{\sigma \lambda}=\left.F^{\sigma \lambda}\right|_{\mathcal{T} \mathcal{X}}=F^{\sigma \lambda}$ очевидно содержит только тривиальную часть (см. замечание 2), так что ядро имеет вид

$$
\operatorname{Ker} \partial_{0}^{m-1}=\left\{J=c_{1} J^{1}+c_{2} J^{2} \mid c_{1}, c_{2} \in \mathbb{R}\right\}
$$

где $J^{1}=\left(u^{1 \lambda}=H^{\lambda}\right)=\mathbf{H}, J^{2}=\left(u^{2 \lambda}=E^{\lambda}-R^{\lambda}\right)=\mathbf{E}-\mathbf{R}$.

В силу замечания 1 отсюда следует, что справедлива

Теорема 5. Линейное пространство может быть представлено в виде

$$
\mathrm{CL}^{2}(\mathcal{T} \mathcal{X})=\left\{\omega=c_{1} \omega^{1}+c_{2} \omega^{2} \mid c_{1}, c_{2} \in \mathbb{R}\right\} \simeq \mathbb{R}_{2},
$$

где базисные законы суть

$$
\omega^{1}=F=\sum_{\lambda}\left(H^{\lambda} d_{\lambda} x-E_{\lambda} \cdot d t \wedge d x^{\lambda}\right)
$$

- тензор электромагнитного поля, записанный как 2-форма, и

$$
\omega^{2}=* F-\sum_{\lambda}\left(R^{\lambda} d_{\lambda} x+S_{\lambda} \cdot d t \wedge d x^{\lambda}\right),
$$

где 2-форма

$$
* F=\sum_{\lambda}\left(E^{\lambda} d_{\lambda} x+H_{\lambda} \cdot d t \wedge d x^{\lambda}\right)
$$

есть сопряженная по Ходжу к форме F (см., например, книгу [11]).

Поясним, что здесь использовано стандартное обозначение

$$
d_{\lambda} x=\frac{1}{2} \sum_{\mu, \nu} e_{\lambda \mu \nu} \cdot d x^{\mu} \wedge d x^{\nu}
$$

так что $d x^{\lambda} \wedge d_{\mu} x=\delta_{\mu}^{\lambda} d^{3} x, d^{3} x=d x^{1} \wedge d x^{2} \wedge d x^{3}$. Напомним еще трехмерные равенства

$$
d\left(\sum_{\lambda} V_{\lambda} d x^{\lambda}\right)=\sum_{\lambda} \operatorname{rot} \mathbf{V}^{\lambda} \cdot d_{\lambda} x, \quad d\left(\sum_{\lambda} V^{\lambda} d_{\lambda} x\right)=\operatorname{div} \mathbf{V} \cdot d^{3} x .
$$


Отметим также, что исходная система уравнений Максвелла (3.22)-(3.25) на языке дифференциальных форм записывается в виде (см., например, книгу [12])

$$
d F=0, \quad d(* F)+\sum_{\lambda} j^{\lambda} \cdot d t \wedge d_{\lambda} x-\varrho d^{3} x=0 .
$$

Таким образом, связи (3.23), (3.25) привели $к$ тому, что у эволючионной системы (3.22), (3.24) наряду с известным стандартным пространством законов сохранения $\mathrm{CL}^{3}(\mathcal{T} \mathcal{X})$ имеется и нетривиальное пространство законов сохранения низшей степени $\mathrm{CL}^{2}(\mathcal{T} \mathcal{X})$.

Благодарности. Работа выполнена при частичной финансовой поддержке РФФИ (гранты № 07-01-00144_а, 09-01-12161-офи-м) и Программы поддержки ведущих научных школ (грант НШ-3224.2008.1).

\section{Список литературы}

[1] Л. В. Овсянников, Групповой анализ дифференииальных уравнений, Наука, М., 1978.

[2] Н. Х. Ибрагимов, Группы преобразований в математической физике, Наука, М., 1983.

[3] П. Олвер, Приложения групп Ли к дифференииальным уравнениям, Мир, М., 1989.

[4] I. S. Krasil'shchik, A. M. Vinogradov, Acta Appl. Math., 15:1-2 (1989), 161-209.

[5] V.V. Zharinov, Lecture Notes on Geometrical Aspects of Partial Differential Equations, Ser. Sov. East Eur. Math., 9, World Sci., Singapore, 1992.

[6] А. М. Виноградов, И. С. Красильщик (ред.), Симметрии и законы сохранения уравнений математической физики, Факториал, М., 1997.

[7] В. В. Жаринов, ТМФ, 68:2 (1986), 163-171.

[8] В. В. Жаринов, Матем. сб., 184:5 (1993), 39-54.

[9] А. М. Виноградов, ДАН СССР, 238:5 (1978), 1028-1031.

[10] T. Tsujishita, Osaka J. Math., 19:2 (1982), 311-363.

[11] Б. А. Дубровин, С.П. Новиков, А.Т. Фоменко, Современная геометрия. Методъ и приложения. Т. 2. Геометрия и топология многообразий, Эдиториал УРСС, М., 2001.

[12] Р. Ботт, Л. В. Ту, Дифференциальные формы в алгебраической топологии, Наука, Физматлит, М., 1989. 